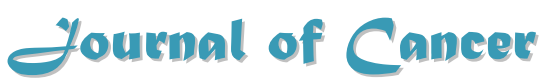

2010; $1: 27-31$

Case Report

(C) Ivyspring International Publisher. All rights reserved

\title{
Ovarian Cancer Metastatic to the Breast Presenting as Inflammatory Breast Cancer: A Case Report and Literature Review
}

\author{
Rebecca L. Klein'1, Amanda R. Brown'1, Christian M. Gomez-Castro², Setsuko K. Chambers³, Janiel M. \\ Cragun $^{3}$, Lauren Grasso-LeBeau ${ }^{4}$, Julie E. Lang ${ }^{1 凶}$
}

1. Department of Surgery, Section of Surgical Oncology, The University of Arizona Cancer Center Tucson, Arizona, USA;

2. Department of Radiology, The University of Arizona Cancer Center Tucson, Arizona, USA;

3. Department of Obstetrics and Gynecology, Section of Gynecologic Oncology, The University of Arizona Cancer Center Tucson, Arizona, USA;

4. Department of Pathology, The University of Arizona Cancer Center Tucson, Arizona, USA.

$\checkmark$ Corresponding author: Julie E. Lang, MD, Section of Surgical Oncology, Arizona Cancer Center, 1515 N. Campbell Avenue, PO Box 245024, Tucson, AZ 85724-5024. Email: jlang@azcc.arizona.edu; Phone: (520) 626-3970; FAX: (520) 626-7785.

Published: 2010.06.02

\begin{abstract}
Background. Primary ovarian carcinoma with metastasis to the breast is rare, with only 39 cases reported in the current literature. Ovarian metastasis to the breast presenting as inflammatory breast carcinoma is even more infrequent, with only 6 cases reported.

Case. We present a patient who developed metastatic inflammatory cancer of the breast from a stage IIIC papillary serous ovarian adenocarcinoma approximately I year after the original diagnosis. Pathologic analysis confirmed the origin of the tumor: a high-grade adenocarcinoma morphologically similar to the previously diagnosed ovarian cancer. In addition, the tumor was strongly positive on immunohistochemistry for CA-125, identical to the ovarian primary. The patient died of diffuse metastasis 5 months after the breast tumor was noted.

Conclusion. Although ovarian metastasis to the breast presenting as inflammatory breast cancer is rare, it should be included in the differential diagnosis for any patient with a personal history of ovarian cancer. Accurate differentiation is necessary because treatment differs significantly for patients with ovarian metastasis to the breast, as compared with patients with primary inflammatory breast cancer. Ovarian metastasis to the breast confers a poor prognosis: patient survival ranged from 3 to 18 months, with a median survival of 6 months after the diagnosis of the breast metastasis.
\end{abstract}

Key words: breast cancer, ovarian cancer, inflammatory breast cancer, ovarian cancer metastasis, breast cancer metastasis

\section{Case Report}

Our patient, a 60-year-old woman, originally presented to her primary care physician for pelvic discomfort in September 2006. At that time, she underwent a pelvic ultrasound as well as a computed tomography (CT) scan of the abdomen and pelvis, which showed a complex ovarian mass. A Pap smear performed one month later was positive for atypical glandular cells suspicious for adenocarcinoma. In November 2006, she underwent a total abdominal hysterectomy with bilateral salpingoophorectomy, omentectomy, and periaortic lymphadenectomy. Upon intraoperative exam, her surgeons noted involvement of the omentum and appendix, as well as studding of the small bowel mesentery and right di- 
aphragm. The pathologic specimen showed extension of the tumor throughout the fallopian tubes, appendix, omentum, and 5 out of 5 positive lymph nodes. The final pathologic diagnosis of the tumor was stage IIIC papillary serous ovarian adenocarcinoma. The patient underwent placement of an intraperitoneal catheter and an intravenous Port-A-Cath for initiation of chemotherapy in December 2006.

The remainder of the patient's past medical history is noncontributory as she was previously in excellent health prior to the diagnosis of ovarian cancer. In addition, she had no known family history of ovarian or breast cancer.

Her initial chemotherapeutic regimen was intraperitoneal and intravenous cisplatin and Taxol. Unfortunately, she suffered a hypersensitivity reaction to the Taxol, and was therefore switched to carboplatin and Abraxane. She received a total of 8 cycles of that combination, with only 1 cycle postponed secondary to neutropenia. Just before the 8th cycle, in order to assess her response to treatment, she underwent a CT scan of the chest, abdomen, and pelvis. The CT scan showed persistent disease around the vaginal cuff. In addition, her CA-125 level, which had previously reached a plateau of $30 \mathrm{U} / \mathrm{ml}$, had risen to $123 \mathrm{U} / \mathrm{ml}$.

Concerned about the possibility of drug-resistant disease, she was evaluated for enrollment in a trial of Avastin and Tarceva. She underwent a new CT scan of the chest, abdomen, and pelvis, in order to obtain baseline data for the trial ( 3 months after her previous CT scan). The new CT scan showed interval development of right axillary lymphadenopathy; the largest lymph node was $1.1 \times 1.8 \mathrm{~cm}$ and suspicion of a new primary breast cancer was raised. We proceeded with breast magnetic resonance imaging (MRI) with gadolinium which showed no suspicious lesions or masses. She therefore continued on Avastin and Tarceva. However, she developed a significant rash in association with these drugs and required dose reduction.

In October 2007, almost a year after her initial ovarian cancer diagnosis, the patient reported the new-onset of right breast edema. Although she had been previously followed for the right axillary lymphadenopathy, she had recently noticed an increase in erythema, thickness, and warmth of the skin of her right breast [Fig 1, 2]. She was treated with a 10-day course of antibiotics, with no change in symptoms. She then underwent an ultrasound of her breast that showed an ill-defined hypoechoic area in the right upper outer quadrant with multiple enlarged lymph nodes. A subsequent mammogram showed scattered fibroglandular densities and an area of architectural distortion with a few small punctate calcifications. Her gynecologic oncologist performed a fine-needle aspiration of the breast, which showed cells consistent with adenocarcinoma. She then underwent a second bilateral breast MRI, which confirmed the presence of an area of heterogeneous enhancement measuring $8 x$ $4 \mathrm{~cm}$, highly suggestive of cancer, with areas suspicious for tumoral extension to the chest wall [Fig 3, 4].

Because of these findings, the patient was referred to a breast surgical oncologist, who performed a punch biopsy of her right breast. Pathologic analysis showed multiple foci of high-grade adenocarcinoma with dermal lymphatic invasion, with morphology similar to that of the previous ovarian cancer [Fig 5]. Furthermore, the breast tissue specimen immunohistochemistry results were positive for CA-125, but negative for estrogen receptor (ER), progestin receptor (PR), and the HER-2/neu oncoprotein. Upon review by our institution's multidisciplinary tumor board, it was concluded that these results were consistent with an ovarian primary tumor.

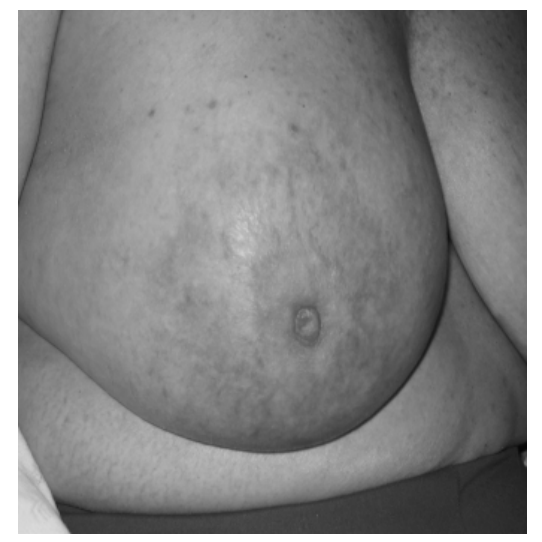

Figure I. Picture demonstrating the inflammatory changes present on initial presentation.

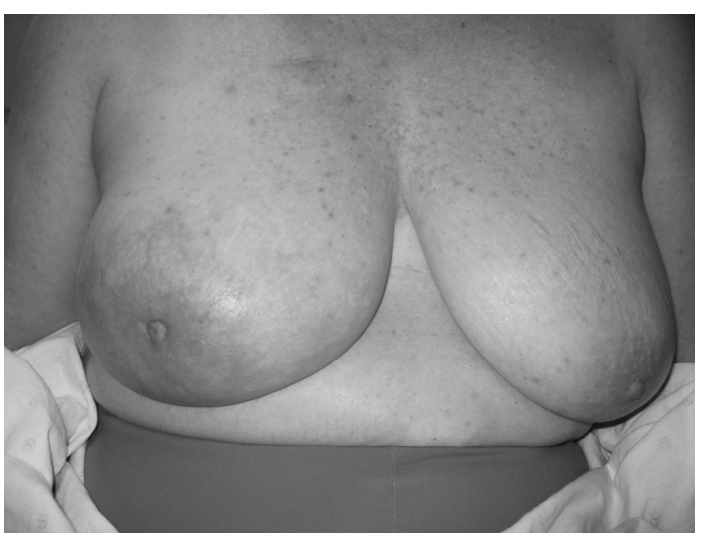

Figure 2. Picture demonstrating the size discrepancy between the two breasts. 


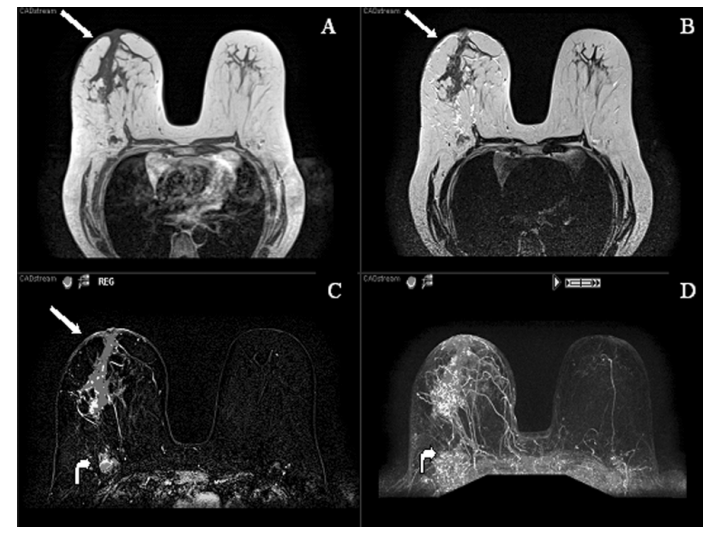

Figure 3. $M R$ images (TI, T2, post-contrast subtracted and MIP) demonstrating a large segmental area of heterogeneous enhancement in the right central breast. There is also abnormal skin enhancement, thickening and edema (straight arrows) consistent with inflammatory changes related to dermal lymphatic involvement. Significant right axillary lymphadenopathy is also present (curved arrow).

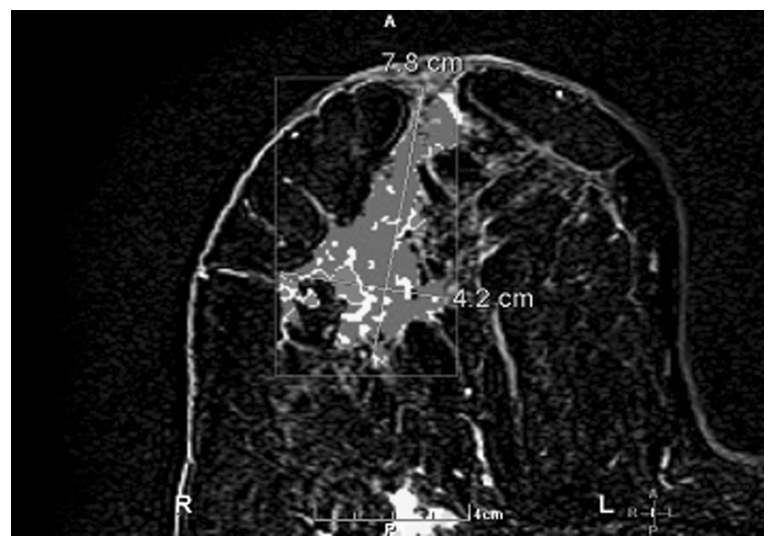

Figure 4. Post contrast subtracted with $C A D$ stream $M R$ image shows a $7.8 \mathrm{~cm} \times 4.2 \mathrm{~cm}$ heterogeneously enhancing mass containing several foci of rapid washout (Type III kinetics) compatible with carcinoma.

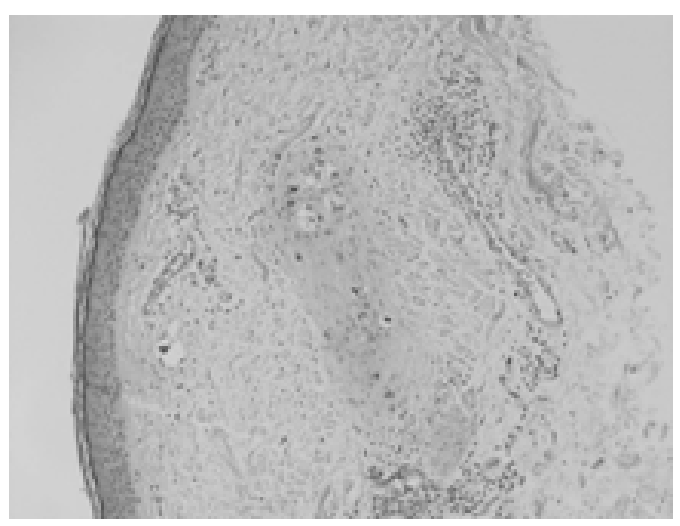

Figure 5. Intermediate power microscopic image depicting metastatic tumor cells involving superficial dermal lymphatics.

\section{Discussion}

Breast cancer is one of the most common primary malignancies in women, yet metastatic tumors to the breast are infrequent, accounting for only $0.5 \%$ to $1.3 \%$ of breast cancer cases [1]. The most common source of metastasis to the breast is a contralateral primary breast tumor, frequently from transthoracic or lymphatic spread. However, hematogenous metastasis from extramammary malignancies has been reported [2]. A study by Hadju and Urban involving 4,051 breast cancer patients found an overall incidence of primary gynecologic cancers metastatic to the breast of $0.17 \%$, with only $0.07 \%$ of metastatic disease originating from a primary ovarian tumor [3]. The first case report of ovarian cancer with metastasis to the breast was in 1907 by Sitzenfrey [4]. To date, a total of only 39 such cases have been reported in the English-language literature [5].

Ovarian metastasis to the breast mimicking primary inflammatory breast carcinoma is even more infrequent, with only 6 previous cases reported [Table 1]. Inflammatory metastasis to a single breast was first reported by Ibach in 1964 [6], followed by 5 other case reports $[2,4,7,8,9]$. Of note, the most recent patients, including ours, all had a diagnosis of stage IIIC papillary serous adenocarcinoma.

In contrast to primary breast tumors, metastasis to the breast generally consists of firm, well-circumscribed, multinodular masses. In addition, the masses are usually superficial and less fixed to surrounding tissues, with the overlying skin generally of normal consistency. The most common form of clinical presentation (in $85 \%$ of patients) was a solitary tumor; only $4 \%$ of patients had diffuse involvement [4]. Furthermore, the most common location was the upper outer quadrant in $62 \%$ of patients [3].

Radiographically, mammographic evaluation can provide additional information. Metastatic tumors to the breast more frequently present as well-circumscribed, non-calcified dense masses. They generally lack spiculation and microcalcifications as well as architectural distortion and other skin changes. However, because of the presence of psammoma bodies associated with some ovarian cancers, microcalcifications can be seen with ovarian metastasis $[3,10]$.

Breast metastasis from a primary ovarian tumor, however, commonly lacks a characteristic pattern, may be morphologically indistinguishable from its primary, and is associated with widespread dissemination. In addition, breast metastasis from a primary ovarian tumor generally is diagnosed an average of 2 
years after the initial diagnosis of ovarian cancer [2]. Our patient developed a markedly diffuse inflammatory process of her right breast with features consistent with inflammatory breast cancer within 1 year after her initial diagnosis.

Histopathological analysis of breast and ovarian tumors can yield similar results. Therefore, accurate differentiation is necessary, because treatment and prognosis differ significantly. The most common histologic variant of ovarian cancer associated with metastatic disease to the breast is papillary serous adenocarcinoma [3]. Immunohistochemistry may provide additional insight into the origin of the tumor. By combining the tumor markers OC125 and OV632, Yamaski et al. found a sensitivity of $86 \%$ and a specificity of $89 \%$ for the diagnosis of metastatic ovarian cancer [10].

Secondary breast involvement from an ovarian tumor suggests widespread dissemination and is associated with a poor prognosis. According to several studies, after the detection of metastatic breast disease secondary to an ovarian primary tumor, survival times ranged from 13 days to 3.5 years [2], with most patients dying within 1 year [11]. Another study found a 1-year survival rate of $40 \%$ for patients with ovarian cancer who also had breast metastasis, as opposed to a 4 -year survival rate of $75 \%$ for patients with primary breast cancer [12]. Inflammatory metastatic disease to the breast also confers a grave prognosis: patient survival ranges from 3 to 18 months (median, 6 months) after diagnosis of the metastasis to the breast [Table 1]. Our patient died of diffuse metastasis 5 months after the breast tumor was noted.

Extramammary tumors should be distinguished from primary breast tumors to avoid any unnecessary surgical procedures. Correct diagnosis is vital: surgical interventions for patients with secondary breast cancers are potentially both diagnostic and palliative. Ovarian metastasis to the breast should be treated as a systemic disease, with appropriate chemotherapeutic agents. Mastectomy of the breast mass is likely best reserved for patients who are unresponsive to systemic therapy and require palliation [11].

Table I. Reported Cases of Ovarian Cancer Metastastic the Breast Presenting as Inflammatory Cancer

\begin{tabular}{|c|c|c|c|c|c|c|}
\hline CASE & AUTHOR & DATE & TYPE OF OVARIAN TUMOR & SURVIVAL ${ }^{a}$ & $\begin{array}{l}\text { INTERVAL TIME TO } \\
\text { DIAGNOSIS }\end{array}$ & THERAPY \\
\hline 1 & Ibach & 1963 & Cyst-adenocarcinoma & 8 months & 2 months & $\begin{array}{l}\text { Chemotherapy (Cyclophos- } \\
\text { phamide) }\end{array}$ \\
\hline 2 & Krishnan & 1980 & Papillary Adenocarcinoma & 6 months & 10 months & $\begin{array}{l}\text { Chemotherapy (Cytoxan, Me- } \\
\text { thotrexate, Leucovorin) and } \\
\text { Radiation }\end{array}$ \\
\hline 3 & Ozguroglu & 1999 & Papillary Serous Adenocarcinoma & 3 months & 2 years & Chemotherapy \\
\hline 4 & Fondrinier & 1999 & $\begin{array}{l}\text { Papillary Serous } \\
\text { Adenocarcinoma }\end{array}$ & 3 months & 3 years & Chemotherapy \\
\hline 5 & $\begin{array}{l}\text { Kayik- } \\
\text { cioglu }\end{array}$ & 2001 & Papillary Serous Adenocarcinoma & 18 months & 18 months & $\begin{array}{l}\text { Chemotherapy (Paclitaxel, Cis- } \\
\text { platin, Anthracycline) }\end{array}$ \\
\hline 6 & Sato & 2007 & $\begin{array}{l}\text { Clear Cell } \\
\text { Carcinoma }\end{array}$ & 10 months & 22 months & Chemotherapy \\
\hline $\begin{array}{l}\text { Current } \\
\text { Case }\end{array}$ & Klein & 2008 & Papillary Serous Adenocarcinoma & 5 months & 12 months & $\begin{array}{l}\text { Chemotherapy (Cisplatin, Gem- } \\
\text { citabine) }\end{array}$ \\
\hline
\end{tabular}

a Time from diagnosis of inflammatory breast lesion to death

${ }^{\mathrm{b}}$ Time from diagnosis of ovarian cancer to breast lesion

\section{Conclusion}

Although ovarian metastasis to the breast presenting as inflammatory breast cancer is rare, it should be included in the differential diagnosis for any patient with a personal history of ovarian cancer. Accurate differentiation is necessary because treatment differs significantly for patients with ovarian metastasis to the breast, as compared with patients with primary inflammatory breast cancer. Ovarian metastasis to the breast confers a poor prognosis: patient survival ranged from 3 to 18 months, with a median survival of 6 months after the diagnosis of the breast metastasis.

\section{Conflict of Interest}

The authors have declared that no conflict of interest exists.

\section{References}

1. Wadhwa J, Dawar R, Kumar L. Ovarian carcinoma metastatic to the breast. Clinical Oncology 1999; 11(6):419-21.

2. Ozguroglu M, Ersavasti G, Ilvan S, et al. Bilateral inflammatory breast metastases of epithelial ovarian cancer. Am J Clin Oncol 1999; 22(4):408-10.

3. Moore D, Wilson D, Hurteau J, et al. Gynecologic cancers metastatic to the breast. J Am Coll Surg 1998; 187:178-81.

4. Kayikçioglu F, Boran N, Ayhan A, Güler N. Inflammatory breast metastases of ovarian cancer: a case report. Gynecologic Oncology 2001; 83(3):613-6. 
5. Baykal C, Tulunay G, Özfuttu A, et al. Breast and ovarian carcinoma in the same patient, metastasis or dual primaries? Turkish Journal of Cancer 2007; 37(1):27-30.

6. Ibach JJr. Carcinoma of the ovary metastatic to the breast. Arch Surg 1964; 88:410-4.

7. Krishnan E, Phillips A, Randell A, et al. Bilateral metastatic inflammatory carcinoma in the breast from primary ovarian cancer. Obstet Gynecol 1980; 55(3 Suppl):94S-6S.

8. Fondrinier E, Gamelin E, Verriele V. Inflammatory breast metastasis from primary ovarian cancer: case report. Eur J Gynaecol Oncol 1999; 20(1):16-7.

9. Sato T, Muto I, Fushiki M, et al. Metastatic breast cancer from gastric and ovarian cancer, mimicking inflammatory breast cancer: report of two cases. Breast Cancer 2008; 15(4):315-20.

10. Yamasaki H, Saw D, Zdanowitz J, Faltz L. Ovarian carcinoma metastasis to the breast: a case report and review of the literature. Am J Surg Pathol 1993; 17(2):193-7.

11. Micha J, Goldstein B, Epstein H, et al. Ovarian cancer metastatic to the breast. Gynecologic Oncology 2006; 102(2):386-90.

12. Loredo D, Powell J, Reed W, Rosenbaum J. Ovarian carcinoma metastatic to breast: a case report and review of the literature. Gynecologic Oncology 1990; 37(3):432-6. 